\title{
A WHITEHEAD PRODUCT FOR TRACK GROUPS II
}

\author{
K.A. HARDIE AND A.V. JANSEN
}

Abstract

Two direct relations are exhibited between the Whitehead product for track groups studied in [4] and the generalized Whitehead product in the sense of Arkowitz. The problem of determining the order of the Whitehead square is posed and some computations given.

\section{Introduction}

Recall that the generalized Whitehead product in the sense of Arkowitz [2] is an operation

$$
\pi(\Sigma A, X) \times \pi(\Sigma B, X) \rightarrow \pi(\Sigma(A \# B), X)
$$

whereas the product studied in [4] (the first paper in this series) is an operation

$$
\pi\left(\Sigma^{m} W, X\right) \times \pi\left(\Sigma^{n} W, X\right) \rightarrow \pi\left(\Sigma^{m+n-1} W, X\right)
$$

that corresponds, under the adjunction isomorphism

$$
\theta: \pi\left(\Sigma^{m} W, X\right) \rightarrow \pi_{m}\left(X^{W}\right),
$$

to the classical Whitehead product of elements of $\pi \cdot\left(X^{W}\right)$.

Following established usage we denote the Arkowitz product and the classical product by square brackets $[-,-]$ and denote the product 0.2 thus: $[-,-]^{W}$.

The key to the new identities is an expression for the universal example class of the product 0.2. Let $\iota_{n n}: S^{m} \rightarrow S^{m} \vee S^{n}, \iota_{n}: S^{n} \rightarrow S^{m} \vee S^{n}$ denote the class of the identity map into the first, respectively second, wedge factor. Identifying $\Sigma^{n} W$ with $S^{n} \# W$ and denoting the identity class $W \rightarrow W$ by $1_{W}$, we can recognize

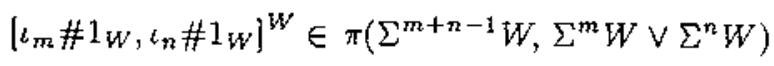

as the universal example class [3] of the product 0.2 .

Then we have the following equality. 
0.4 Proposition. $\left[\iota_{m} \# 1_{W}, \iota_{n} \# 1_{W}\right]^{W}=\left[\iota_{m}, \iota_{n}\right] \# 1_{W}$

Now let $\alpha \in \pi\left(\Sigma^{m} W, X\right), \beta \in \pi\left(\Sigma^{n} W, X\right)$ be given classes, let $\chi: W \rightarrow$ $W \# W$ denote the diagonal class and let

$$
\rho: S^{m+n-1} \# W \# W \rightarrow S^{1} \# S^{m-1} \# W \# S^{n-1} \# W
$$

be the natural homeomorphism permuting the smash factors.

The following result shows that the track group product and the Arkowitz product are related via the diagonal class.

0.5 Proposition. $[\alpha, \beta]^{W}=[\alpha, \beta] \circ \rho \circ \Sigma^{m+n-1} \chi$.

An obvious consequence of Proposition 0.5 is that nontriviality of $[\alpha, \beta]^{W}$ implies nontriviality of $[\alpha, \beta]$. We remark that in this way, through the methods of [4], many generalized Whitehead products can be shown to be nontrivial.

It may be recalled from [4] (or deduced from Proposition 0.5) that $[\alpha, \beta]^{W}=0$ whenever $W$ itself is a suspension. In consequence it may be useful to study the behaviour of the product in the case of $W$ a product of suspensions. The following identity applies when $W=U \times V$ with $U$ and $V$ arbitrary.

0.6 Proposition. Let $\alpha \in \pi\left(\Sigma^{m} U, X\right), \beta \in \pi\left(\Sigma^{n} V, X\right)$, let pru and $p r_{V}$ denote the projections from $U \times V$ on to the factor spaces and let $q: U \times V \rightarrow$ $U \# V$ denote the smash identification. Then

$$
\begin{aligned}
& {\left[\alpha \circ \Sigma^{m} p r_{U}, \beta \circ \Sigma^{n} p r_{V}\right]^{U \times V}=[\alpha, \beta] \circ \rho \circ \Sigma^{m+n-1} q} \\
& \text { in } \pi\left(\Sigma^{m+n-1}(U \times V), X\right) .
\end{aligned}
$$

Proposition 0.6 can be regarded as a generalization to the case $m \geq 1$, $n \geq 1$ of Arkowitz's Theorem 2.4 [2] which establishes the equivalence of two alternative definitions of his generalized Whitehead product.

Proposition 0.4 is the universal example case of the following result.

0.7 Corollary. Let $\alpha \in \pi_{m}(X), \beta \in \pi_{n}(X)$. Then

$$
\left[\alpha \# 1_{W}, \beta \# 1_{W}\right]^{W}=[\alpha, \beta] \# 1_{W} \text { in } \pi\left(\Sigma^{m+n-1} W, X \# W\right) .
$$

In section 3 we consider some instances of the Whitehead square

$$
\gamma=\left[\Sigma^{n} 1_{W}, \Sigma^{n} 1_{W}\right]^{W} \in \pi\left(\Sigma^{2 n-1} W, \Sigma^{n} W\right) .
$$

In the case $W=S^{0}, \gamma$ is the classical Whitehead square so that we have $\gamma=0$ if and only if $n=1,3,7[1]$. It would be interesting to have corresponding information for $W$ an arbitrary non-suspension. However for the moment this seems to be out of reach. Instead we enquire when the order of $\gamma$ is infinite and obtain a necessary condition which we show can occur.

It is a pleasure to acknowledge that an earlier version of this paper was read by Howard Marcum whose comments and suggestions gave rise to a number of improvements. 


\section{Universal examples}

The universal example class [ 3 ] for the operation 0.2 is obtained by applying the operation to the classes

$$
\Sigma^{m} W \rightarrow \Sigma^{m} W \vee \Sigma^{n} W, \quad \Sigma^{n} W \rightarrow \Sigma^{m} W \vee \Sigma^{n} W
$$

of identity maps into the first and second wedge factors. Since $\Sigma^{m} W \vee \Sigma^{n} W=$ $\left(S^{m} \vee S^{n}\right) \# W$, with the conventions stated in the introduction the classes 1.1 become $\iota_{m} \# 1_{W}$ and $\iota_{n} \# 1_{W}$ respectively. Hence the desired universal class is

$$
\theta^{-1}\left[\theta\left(\iota_{m} \# 1_{W}\right), \theta\left(\iota_{n} \# 1_{W}\right)\right] \in \pi\left(\Sigma^{m+n-1} W,\left(S^{m} \vee S^{n}\right) \# W\right)
$$

Now we recall that the isomorphism $\theta$ expresses the situation that the functor $-\# W$ is left adjoint to $(-)^{W}$. The unit of the adjunction is a natural transformation that assigns to each space $X$ a class

$$
\eta X: X \rightarrow(X \# W)^{W}
$$

with a certain universal property. Consider the diagram

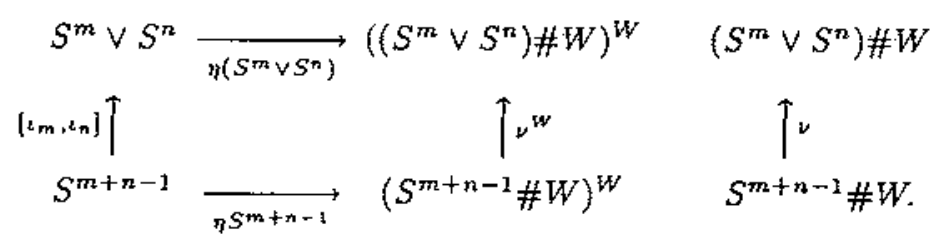

Since $\left[t_{m}, t_{n}\right]$ is the universal example class for the (classical) Whitehead product we have

$$
\eta\left(S^{m} \vee S^{n}\right) \circ\left[\iota_{m}, \iota_{n}\right]=\left[\theta\left(\iota_{m} \# 1_{W}\right), \theta\left(\iota_{n} \# 1_{W}\right)\right] .
$$

By the universal property of $\eta$ the dotted arrow $\nu=\theta^{-1}\left(\eta\left(S^{m} \vee S^{n}\right) \circ\left[\iota_{m}, \iota_{n}\right]\right)$ is the unique class making the rectangle commutative. However by naturality of $\eta$ if we set $\nu=\left[\iota_{m}, \iota_{n}\right] \# 1_{W}$ the rectangle is commutative. This completes the proof of Proposition 0.4.

1.2 Remark. The reader will note that the argument above does not depend on the intrinsic nature of the universal example class $\left[\iota_{m}, \iota_{n}\right]$.

\section{Joins}

The universal example class for the generalized Whitehead product 0.1 is a certain element of

$$
\pi(\Sigma(A \# B), \Sigma A \vee \Sigma B)
$$


An annoying technical feature of the definition and resulting theory is the following consideration. In order to establish properties of the product it is desirable to realize the class as a natural transformation at the level of maps. Unfortunately no such natural transformation exists. It seems that one has to be content with a pair of natural transformations, in opposite directions

$$
\Sigma(A \# B) \stackrel{\mu^{\prime}}{\leftarrow} A * B \rightarrow \Sigma A \vee \Sigma B
$$

emanating from $A * B$, the join of $A$ and $B$, with the property that the reverse arrow $\mu^{\prime}$ is a homotopy equivalence for each $A$ and $B$. Recall that $A * B$ is the space obtained from $A \times B \times I$ by factoring out the relations $(a, b, 0) \sim\left(a, b^{\prime}, 0\right)$ and $(a, b, 1) \sim\left(a^{\prime}, b, 1\right)$ for all $a, a^{\prime} \varepsilon A$ and $b, b^{\prime} \in B$. The base point of $A * B$ is usually chosen to be $(*, *, 1 / 2)$. This feature of the join is undotabtedly very inconvenient. For example it has the consequence that the factors $A$ and $B$ are not automatically embedded in $A * B$ since the obvious maps

$$
a \rightarrow(a, *, 0) \quad b \rightarrow(*, b, 1)
$$

do not preserve base points. More serious for our immediate purpose is the consideration that the base point hinders smooth interaction with the smash product. To remedy the situation we work rather with $A \star B$, obtained from $A * B$ by further identifying all points $(*, *, t)(t \varepsilon I)$. The equivalence class of $(a, b, t)$ as a point of $A \star B$ will be denoted $[a, b, t]$. Note that the Whitehead product map

$$
\nu: A \star B \rightarrow \Sigma A \vee \Sigma B
$$

(corresponding to the composite $h \nu$ in [2]) is given by the formula

$$
\nu[a, b, t]= \begin{cases}(*,(b, 1-2 t)) & (0 \leq t \leq 1 / 2) \\ ((a, 2 t-1), *) & (1 / 2 \leq t \leq 1) .\end{cases}
$$

If we also let $\mu^{\prime}: A \star B \rightarrow \Sigma(A \# B)$ be given by $\mu^{\prime}[a, b, t]=(a, b, t)$ then $\mu^{\prime}$ is a homotopy equivalence with homotopy inverse $\mu$ (say). The universal example class for the generalized Whitehead product 0.1 as defined in [2] is then

$$
\{\nu \mu\}\left[\iota_{\Sigma A}, \iota_{\Sigma \beta}\right] \in \pi(\Sigma(A \# B), \Sigma A \vee \Sigma B) \text {. }
$$

Identifying $\Sigma A$ with $S^{1} \# A$, we have $(\Sigma A \vee \Sigma B) \# W=S^{1} \# A \# W \vee S^{1} \# B \# W=$ $\Sigma(A \# W) \vee \Sigma(B \# W)$. We claim

\subsection{Proposition.}

$$
\left[\iota_{\Sigma}, \iota \Sigma B\right] \# I_{W}=\left[\iota_{\Sigma(A \# W), \iota \Sigma(B \# W)}\right] \circ \rho \circ\left(1_{\Sigma(A \# B)} \# \chi\right),
$$

where $\rho: S^{1} \# A \# B \# W \# W \rightarrow S^{3} \# A \# W \# B \# W$ interchanges the central smash factors $B$ and $W$.

Proof: Consider

$$
\sigma:(A \star B) \# W \rightarrow(A \# W) \star(B \# W)
$$


given by the formula

$$
\sigma([a, b, t], w)=[(a, w),(b, w), t] .
$$

Note that $\sigma$ respects the $\star$ identifications and so defines a (pointed, continuous) map. We remark that this would not be the case if $\star$ were replaced by the classical join $\star$. Then there is a commutative diagram (of solid arrows)

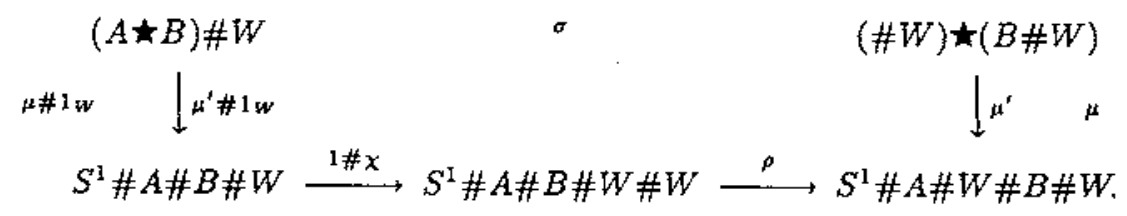

Using 2.1 and 2.4 it can be checked that

$$
\nu \sigma=\nu \# 1 \mathrm{~W} .
$$

The required equality is then a consequence of 2.2. Combining Proposition 0.6 with the relevant special case of Proposition 2.3 now yields Proposition 0.5.

Proposition 0.6 can be obtained from the case $W=U \times V$ of 2.4 after some further analysis. In view of the commutativity of the diagram

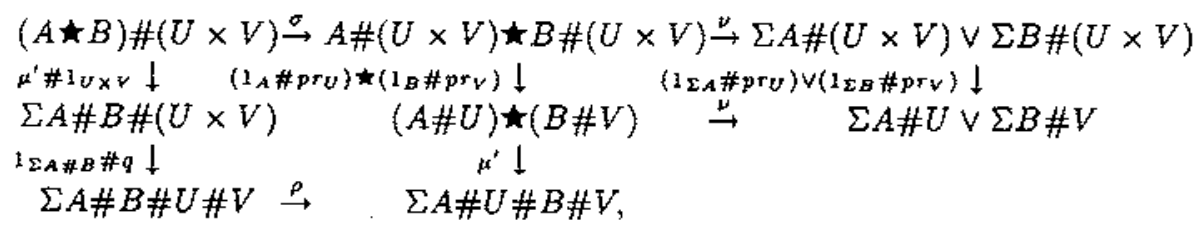

we have the following equality

$$
\begin{aligned}
& \left(\left(1_{\Sigma A} \# p r_{U}\right) \vee\left(1_{\Sigma B} \# p r_{V}\right)\right) \circ\left[\sum_{\left.\iota_{\Sigma A}, \iota_{\Sigma B}\right] \# I_{U \times V}}\right. \\
& =\left[\iota_{\Sigma A \# U}, \iota_{\Sigma B \# V}\right] \circ \rho \circ 1_{\Sigma A \# B} \# q .
\end{aligned}
$$

The case $A=S^{m-1}, B=S^{n-1}$ of 2.5 can be expressed using Proposition 0.4 as

$$
\begin{aligned}
& \left(\Sigma^{m} p_{U} \vee \Sigma^{n} p r_{V}\right) \circ\left\{\iota_{m} \# 1_{U \times V}, \iota_{n} \# 1_{U \times V}\right]^{U \times V} \\
& =\left[\iota \Sigma^{m} U, \iota \Sigma^{n} V\right] \circ \rho \circ \Sigma^{m+n-1} q,
\end{aligned}
$$

which is simply the assertion of Proposition 0.6 in the case of the universal example. 


\section{Whitehead squares}

In this section we give attention to certain instances of the Whitehead square

$$
\gamma=\left[\Sigma^{n} 1_{W}, \Sigma^{n} 1 W\right]^{W} \in \pi\left(\Sigma^{2 n-1} W, \Sigma^{n} W\right)
$$

considering in particular the following.

Whitehead square problem. Given a non-suspension space $W$, determine $0(\gamma)$, the order of $\gamma$.

In view of Corollary 0.7 we have

$$
\gamma=\left[\iota_{n}, \iota_{n}\right] \# 1_{W},
$$

where $\left[t_{n}, t_{n}\right] \in \pi_{2 n-1}\left(S^{n}\right)$ is the classical Whitehead square. Hence full information is available in the simplest case: $W=S^{0}$. According to Adams [1], $\gamma=0$ if and only if $n=1,3,7$; otherwise $0(\gamma)=2$ ( $n$ odd $), 0(\gamma)=\infty$ ( $n$ even).

Suppose next that $W$ is a complex with two cells: $W=S^{m} U_{a} e^{r+1}$, where $\alpha \in \pi_{r}\left(S^{n}\right)$ is not a suspension class, so that $r \geq 2 m-1$. We have the following crude result.

3.3 Theorem. If $0(\gamma)=\infty$ then $r=2 m-1, m+n$ is even and $n \neq 1,3,7$.

Proof: $n=1,3,7$ imply $\gamma=0$, so these may be excluded. Consider the Puppe sequence at $\Sigma^{n} W$ of the class $\Sigma^{2 n-1} \alpha$ :

$$
\begin{aligned}
& \cdots \rightarrow \pi\left(S^{2 n+r}, \Sigma^{n} W\right) \rightarrow \pi\left(\Sigma^{2 n-1} W, \Sigma^{n} W\right) \rightarrow \pi\left(S^{m+2 n-1}, \Sigma^{n} W\right) \rightarrow \\
& \rightarrow \pi\left(S^{2 n+r-1}, \Sigma^{n} W\right) .
\end{aligned}
$$

Since $\pi\left(S^{m+2 n-1}, \Sigma^{n} W\right)$ lies in the stable range and $\gamma$ is annihilated by suspension, $O(\gamma)=\infty$ implies the existence of an element of infinite order in $\pi_{2 n+r}\left(\Sigma^{n} W\right)$. Since $\Sigma^{n} W=S^{n+m} \cup e^{n+r+1}$, we may consider the relative homotopy sequence

$$
\cdots \rightarrow \pi_{2 n+r}\left(S^{m+n}\right) \rightarrow \pi_{2 n+r}\left(\Sigma^{n} W\right) \rightarrow \pi_{2 n+r}\left(\Sigma^{n} W, S^{m+n}\right) .
$$

Now we have $\pi_{2 n+r}\left(\Sigma^{n} W, S^{m+n}\right) \approx \pi_{2 n+r}\left(S^{n+r+1}\right)$, by [5, VII, Theorem 7.12]. Hence $\pi_{2 n+r}\left(\Sigma^{n} W, S^{n+1}\right)$ is a finite group, being isomorphic to the stable $(n-1)$-stem, with $n>1$. It follows that $\pi_{2 n+r}\left(S^{m+n}\right)$ has an element of infinite order. But this is possible only if $m+n$ is even and $2 n+r=2(m+n)-1$, i.e. $r=2 m-1$.

There are spaces $W$ for which a converse of 3.3 holds:

3.4 Proposition. If $W=S_{2}^{m}$ ( $m$ even), the $2 m$-skeleton of the James reduced product $S_{\infty}^{m}$, and if $n$ is even then $0(\gamma)=\infty$.

We require the following lemma. 
3.5 Lemma. The diagonal $\chi: S_{2}^{m} \rightarrow S_{2}^{m} \# S_{2}^{m}$ ( $m$ even) induces a nontrivial homomorphism of the $2 m$-dimensional integral cohomology groups.

Proof: $\chi$ can be factored $S_{2}^{m} \stackrel{\Delta}{\rightarrow} S_{2}^{m} \times S_{2}^{m} \stackrel{\theta}{\rightarrow} S_{2}^{m} \# S_{2}^{m}$ through the shrinking map $\theta$. In dimension $2 m, \theta^{*}$ is an isomorphism on to a direct summand containing the product of the generators in dimension $m$ of the factor spaces. $\triangle^{*}$ sends this product to the cup square of the generator of $H^{m}\left(S_{2}^{m}\right)$, which is non-trivial.

Proof of 3.4: Suppose, for some integer $r$, that $r \gamma=\left[r \Sigma^{n} 1_{W}, \Sigma^{n} 1_{W}\right]^{W}=0$. Then by $\left[4\right.$, Theorem 2.4] there exists $c\left(r \Sigma^{n} 1_{W}, \Sigma^{n} 1_{W}\right) \subseteq \pi\left(\Sigma^{2 n+1} W, \Sigma^{n+1} W\right)$, a coset of the suspension subgroup, with the property that

$H_{C}\left(r \Sigma^{n} 1_{W}, \Sigma^{n} 1_{W}\right)=r \Sigma^{2 n+1} \chi$ in $\pi\left(\Sigma^{2 n+1} W, \Sigma^{2 n+1} W \# W\right)$, where $H$ refers to a version of the Hopf James invariant homomorphism. It follows from Lemma 3.5 the $r \sum^{2 n+1} \chi$ is an element of infinite order and hence that the coset $c\left(r \Sigma^{n} 1_{W}, \Sigma^{n} 1_{W}\right)$ is of infinite order. We produce a contradiction by arguing that $\pi\left(\Sigma^{2 n+1} W, \Sigma^{n+1} W\right)$ can in this case have no element of infinite order in the cokernel of suspension. Since $\Sigma S_{2}^{m} \cong S^{m+1} \vee S^{2 m+1}$, we have

$$
\begin{aligned}
& \pi\left(\Sigma^{2 n+1} W, \Sigma^{n+1} W\right) \approx \pi_{2 m+2 n+1}\left(S^{m+n+1}\right) \oplus \pi_{2 m+2 n+1}\left(S^{2 m+n+1}\right) \\
& \oplus \pi_{m+2 n+1}\left(S^{m+n+1}\right) \oplus \pi_{m+2 n+1}\left(S^{2 m+n+1}\right) .
\end{aligned}
$$

Since $m+n+1$ is odd, none of the summands has an element of infinite order, excepting only $\pi_{m+2 n+1}\left(S^{2 m+n+1}\right)$ in the case $m=n$. However it is easily seen that this summand with $m=n$ belongs to the suspension subgroup.

3.6. Remark. With $W=S_{2}^{m}$, Proposition 3.4 does not determine $0(\gamma)$ when $n$ is odd, $n \neq 1,3,7$. Since in this case $O\left(\left[t_{n}, t_{n}\right]\right)=2$, we know at least that 2 divides $0(\gamma)$.

3.7. Remark. For the space $K^{\prime}(Z / t, 1)=S^{1} U_{t} e^{2}$, we can conclude from Theorem 3.3 that $0(\gamma)$ is finite for all even $n$.

Acknowledgements The authors acknowledge a grant to the Topology Research Group from the Foundation for Research Development of the South African Council for Scientific and Industrial Research.

\section{References}

1. J.F. ADAMS, On the non-existence of elements of Hopt invariant one, Ann. of Math. (2) 72 (1960), 20-104.

2. M. ARKowITz, The generalized Whitehead product, Pacific J. Math. 12 (1962), 7-23.

3. A.L. BLAKERS, W.S. MASSEY, Products in homotopy theory, Ann. of Math. 58 (1953), 295-324. 
4. K.A. HARDIE, A.V. JANSEN, A Whitehead product for track groups, Proceedings of the Conference on Algebraic Topology (1986), Arcata. (to appear).

5. G.W. WhiteheAD, "Elements of Homotopy Theory," Springer Verlag, 1978 .

\author{
Department of Mathematics \\ University of Cape Town \\ Rondebosch 7700 \\ SOUTH AFRICA
}

Rebut el 25 d'Octubre de 1988 